\title{
Un abordaje genómico del alcoholismo
}

\section{A genomic approach to alcoholism}

\author{
Javier Costas* \\ *Grupo de Xenética Psiquiátrica, Hospital Clínico Universitario de Santiago de Compostela, Instituto de Investigación \\ Sanitaria de Santiago (IDIS), Servizo Galego de Saúde (SERGAS), España
}

$\mathrm{L}$ as tecnologías genómicas basadas en chips de ADN (micromatrices) permiten el análisis simultáneo de cientos de miles de polimorfismos de un único nucleótido (SNP, single nucleotide polymorphisms), lo que ha revolucionado los estudios de asociación caso-control. Se ha pasado de estudios de genes candidatos, según hipótesis etiopatogénicas previas, a estudios de asociación de genoma completo (GWAS, genome-wide association studies), que analizan la gran mayoría de la variación común existente a lo largo del genoma. El impacto sobre el conocimiento de las bases genéticas de predisposición a enfermedades multifactoriales complejas ha sido substancial.

Los trastornos psiquiátricos no han quedado al margen de estos avances, destacando la esquizofrenia, con 37.000 casos y 113.000 controles analizados mediante GWAS por el Psychiatric Genomics Consortium (PGC) (Schizophrenia Working Group PGC, 2104). En otros trastornos psiquiátricos, incluyendo dependencia de alcohol, los tamaños muestrales de los GWAS son mucho más modestos. Los estudios pioneros en esquizofrenia están mostrando las posibles aportaciones de los GWAS, que se van confirmando en otros trastornos. La más obvia es la detección de variantes comunes asociadas al trastorno bajo estudio, empleando el criterio establecido de significación genómica $\left(p<5 \times 10^{-8}\right.$, equivalente a una corrección de Bonferroni para un millón de pruebas). En dependencia de alcohol, los GWAS han confirmado la implicación ya conocida de $A L D H 2$ o la familia de $A D H$ y han identificado unos pocos genes adicionales, como PECR o NKAIN1-SERINC2 (Frank et al., 2012; Treutlein et al., 2009; Zuo et al., 2013). La experiencia en otros trastornos complejos indica que estos estudios, que analizan menos de 2.000 pacientes y similar número de controles en la fase de GWAS, tienen una potencia muy reducida para identificar SNP de susceptibilidad, pues su efecto individual es muy pequeño (odds ratio $<1,25$ ). Así, en esquizofrenia, se ha pasado de identificar como máximo tres asociaciones significativas independientes en análisis con tamaños muestrales similares a los empleados actualmente en dependencia de alcohol a 108 en el último GWAS del PGC, referido anteriomente (Schizophrenia Working Group PGC, 2104; Stefansson et al., 2009). Por tanto, los resultados significativos a nivel genómico representan sólo la punta del iceberg. Por ejemplo, el gen SLC39A8, inicialmente asociado a esquizofrenia en un estudio liderado por nuestro grupo basado en 4.545 pacientes y 15.575 controles $\left(p=2,7 \times 10^{-6}\right)$, llegó al nivel de significación genómico en el mega-GWAS del PGC $\left(p=7,98 \times 10^{-15}\right)$ (Carrera et al., 2012; Schizophrenia Working Group PGC, 2104). 
Dado que numerosas variantes de susceptibilidad no alcanzan significación a nivel genómico, el siguiente paso lógico es la detección de grupos de genes funcionalmente relacionados que estén representados en exceso entre los valores más significativos de un GWAS. A modo de ejemplo, Biernacka et al. (2013) identificaron una posible asociación (pendiente de confirmación en nuevas muestras) con la vía de síntesis y degradación de cuerpos cetónicos. El consumo excesivo de alcohol puede incrementar los niveles de cuerpos cetónicos en sangre, llegando a originar cetoacidosis alcohólica. La cetoacidosis cursa con síntomas como nauseas, vómitos, dolores abdominales, etc., que podrían causar aversión hacia el consumo excesivo de alcohol.

Además de avanzar en la comprensión de las bases biológicas que predisponen a la aparición de un trastorno, los GWAS podrían aplicarse para estimar el riesgo genético individual a sufrir el trastorno. Esta estima tiene una clara limitación, ya que está basada sólo en variantes comunes (algo que podrá paliarse a medida que los estudios de secuenciación de nueva generación empiecen a identificar variantes raras de mayor efecto). Además, el riesgo genético sólo explica una parte del riesgo total. En el caso de alcoholismo, se estima que los factores genéticos explican un $40-60 \%$ de la variación poblacional en riesgo (heredabilidad). A pesar de estas limitaciones, los datos en esquizofrenia (heredabilidad $\sim 65 \%$ ) sugieren que el cálculo del riesgo genético individual a partir de datos de GWAS puede ser útil en la estratificación de grupos de riesgo. Así, el decil de mayor riesgo presenta una odds ratio de entre 8 y 20 en comparación con el de menor riesgo (Schizophrenia Working Group PGC, 2014).

Actualmente, la estima del riesgo poligénico se realiza de una forma simple. A partir de datos de GWAS de una muestra de descubrimiento, se genera un modelo de riesgo que incluya todos los SNP independientes por debajo de un umbral de significación poco estricto y su efecto (logaritmo de odds ratio). Se contabiliza en cada individuo de una muestra diana el número de alelos de riesgo para cada SNP del modelo, ponderados por su efecto. La suma de este valor para todos los SNP considerados constituye la puntuación de riesgo poligénico de un individuo diana. Frank et al. (2012) fueron los primeros en aplicar este método a dependencia de alcohol, a partir de 1.333 casos y 2.168 controles divididos al azar en muestras de descubrimiento y diana de igual tamaño. Usando un umbral de significación $p<0,5$ en el GWAS de la muestra de descubrimiento (lo que equivalió a analizar los $~ 84.000$ SNP independientes más significativos), encontraron una diferencia en la puntuación del riesgo poligénico entre casos y controles en la muestra diana en la dirección esperada $\left(p=1,28 \times 10^{-6}\right)$. Empleando toda la muestra como muestra de descubrimiento, también encontraron diferencias significativas cuando las muestras diana provenían de otros GWAS previos.
Entre los SNP incluidos en el cálculo del riesgo poligénico habrá tanto SNP con efecto real como SNP no relacionados con el trastorno, que generarán ruido. Por tanto, hay un amplio margen de mejora en la estima incrementando el tamaño de la muestra de descubrimiento, lo que reducirá el error de muestreo en la elaboración del modelo de riesgo poligénico. Otra opción de mejora es la priorización de los SNP del modelo según criterios biológicos adicionales a priori, como predicción de funcionalidad o resultados de GWAS en fenotipos relacionados. De hecho, empleando muestras de descubrimiento y diana de fenotipos distintos, se ha comprobado susceptibilidad genética compartida (Cross-Disorder Group PGG, 2013). Este tipo de análisis permitirán entender mejor la patología dual.

Por último, la constatación de que existen personas más susceptibles a convertirse en drogodependientes desde el punto de vista biológico podrá tener relevancia tanto en la reducción del estigma asociado a las personas alcohólicas como en la actitud de los adolescentes y jóvenes frente al alcohol. Diversos estudios indican que existe interés en una potencial prueba genética del riesgo individual a padecer dependencia de alcohol y que la creencia de una predisposición genética individual elevada constituye un incentivo para la adopción de cambios en el patrón de bebida (Dar-Nimrod, Zuckerman y Duberstein, 2013; Scott et al., 2014).

En resumen, los GWAS han demostrado que la predisposición genética a sufrir alcoholismo es debida a múltiples genes con un efecto individual muy pequeño. El estudio de estos genes tendrá consecuencias a medio plazo tanto incrementando la comprensión de los mecanismos biológicos implicados en susceptibilidad a alcoholismo como permitiendo la estratificación de sujetos en función de su riesgo genético a padecer alcoholismo, lo cual constituye un paso importante de cara a la prevención selectiva. Por ejemplo, la estratificación de adolescentes con un patrón de consumo de riesgo o con conductas problemáticas (actos delictivos, violencia) basado en pruebas genéticas podría permitir intervenciones más focalizadas. Dada la gravedad del problema, con más de 25.000 personas demandando tratamiento frente a alcoholismo en España anualmente (según el Observatorio español de la droga y las toxicomanías) cualquier aproximación que consiga paliarlo debe ser bienvenida. En este sentido, el abordaje de las adicciones en los próximos años deberá prestar atención a los avances generados empleando tecnologías genómicas, dada su potencial utilidad. Para que esta potencialidad se manifieste, será imprescindible una colaboración multidisciplinar.

\section{Conflicto de intereses}

El autor declara que no existe ningún conflicto de intereses en este trabajo. 


\section{Referencias}

Biernacka, J. M., Geske, J., Jenkins, G. D., Colby, C., Rider, D. N., Karpyak, V. M., . . . Fridley, B. L. (2013). Genome-wide gene-set analysis for identification of pathways associated with alcohol dependence. International Journal of Neuropsychopharmacology, 16, 271-278. doi: $10.1017 / \mathrm{s} 1461145712000375$.

Carrera, N., Arrojo, M., Sanjuan, J., Ramos-Rios, R., Paz, E., Suarez-Rama, J. J., . . . Costas, J. (2012). Association Study of Nonsynonymous Single Nucleotide Polymorphisms in Schizophrenia. Biological Psychiatry, 71, 169177. doi: 10.1016/j.biopsych.2011.09.032.

Cross-Disorder Group of the Psychiatric Genomics Consortium (2013). Identification of risk loci with shared effects on five major psychiatric disorders: a genome-wide analysis. Lancet, 381, 1371-1379. doi: 10.1016/S01406736(12)62129-1.

Dar-Nimrod, I., Zuckerman, M. y Duberstein, P. R. (2013). The effects of learning about one's own genetic susceptibility to alcoholism: a randomized experiment. Genetics in Medicine, 15, 132-138. doi: 10.1038/gim.2012.111.

Frank, J., Cichon, S., Treutlein, J., Ridinger, M., Mattheisen, M., Hoffmann, P., . . Rietschel, M. (2012). Genome-wide significant association between alcohol dependence and a variant in the $\mathrm{ADH}$ gene cluster. Addiction Biology, 17, 171-180. doi: 10.1111/j.1369-1600.2011.00395.x.

Schizophrenia Working Group of the Psychiatric Genomics Consortium (2014). Biological insights from 108 schizophrenia-associated genetic loci. Nature, 511, 421427. doi: 10.1038/nature13595.

Scott, D. M., Nwulia, E., Kwagyan, J., Cain, G., Marshall, V. J., Kalu, N., . . . Taylor, R. E. (2014). Genetic Testing for the Susceptibility to Alcohol Dependence: Interest and Concerns in an African American Population. Genet Test Mol Biomarkers, 18, 538-545. doi: 10.1089/ gtmb.2013.0417.

Stefansson, H., Ophoff, R. A., Steinberg, S., Andreassen, O. A., Cichon, S., Rujescu, D., . . Collier, D. A. (2009). Common variants conferring risk of schizophrenia. $\mathrm{Na}$ ture, 460, 744-747. doi: 10.1038/nature08186.

Treutlein, J., Cichon, S., Ridinger, M., Wodarz, N., Soyka, M., Zill, P., . . Rietschel, M. (2009). Genome-wide association study of alcohol dependence. Arch Gen Psychiatry, 66, 773-784. doi: 10.1001/archgenpsychiatry.2009.83.

Zuo, L., Wang, K., Zhang, X. Y., Krystal, J. H., Li, C. S., Zhang, F., . . . Luo, X. (2013). NKAIN1-SERINC2 is a functional, replicable and genome-wide significant risk gene region specific for alcohol dependence in subjects of European descent. Drug Alcohol Depend, 129, 254-264. doi: 10.1016/j.drugalcdep.2013.02.006. 